Supporting Information for

\title{
The First Regiospecific, Enantiospecific Total Synthesis of 6-Oxoalstophylline and an Improved Total Synthesis of Alstonerine and Alstophylline as well as the Bisindole Alkaloid Macralstonine
}

\author{
Xuebin Liao, Hao Zhou, Xiangyu Z. Wearing, Jun Ma and James M. Cook* \\ Department of Chemistry, University of Wisconsin-Milwaukee, \\ Milwaukee, WI 53201 \\ capncook@uwm.edu
}

\section{Experimental Section.}

Melting points were taken on a Thomas-Hoover melting point apparatus or an Electrothermal model IA8100 digital melting point apparatus and are uncorrected. Microanalyses were performed on a Perkin-Elmer 240C carbon, hydrogen, and nitrogen analyzer. All samples submitted for $\mathrm{CHN}$ analyses were first dried under high vacuum for a minimum of six hours using a drying pistol with isopropyl alcohol as the solvent with phosphorus pentoxide in the drying bulb. Proton and carbon high resolution nuclear magnetic resonance spectra were obtained on a bruker 300-MHzNMR spectrometer or GE 500-MHz NMR spectrometer. The low resolution mass spectra (EI/CI) were obtained on a Hewlett-Packard 5985B gas chromatographymass spectrometer, while high resolution spectra were recorded on a VG Autospec (Manchester, England) mass spectrometer. Infra-red spectra were recorded on a Nicolet MX-1 FT-IR or a Perkin Elmer 1600 Series FT-IR spectrometer. 
Analytical thin layer chromatography plates used were E. Merck Brinkmann UV-active silica gel (Kieselgel $60 \mathrm{~F} 254$ ) on plastic. Silica gel 60A, grade 60 for flash and gravity chromatography, was purchased from E. M. Laboratories.

Alkaloids were visualized with a saturated solution of ceric ammonium sulfate in $50 \%$ sulfuric acid. Methanol $(\mathrm{MeOH})$ and ethanol $(\mathrm{EtOH})$ were dried by distillation over magnesium metal and iodine. Tetrahydrofuran (THF), benzene, toluene, dioxane, and diethyl ether were dried by distillation from sodium benzophenone ketyl. Dichloromethane was dried over $\mathrm{MgSO}_{4}$ and then distilled over $\mathrm{CaH}_{2}$. Triethylamine, diisopropylamine and pyridine were dried by distillation over $\mathrm{KOH}$.

\section{Sodium Borohydride Mediated Reduction of 3-Ethylidene-10-methoxy-12-methyl-} 1,3,4,7,12,12b- hexahydro-13-hydroxy methyl-2H,6H-2,6-methano-indolo [2,3- $\alpha]-$ quinolizine-13-carboxaldehyde 7 to Provide the (+)-3-Ethylidene-12-methyl-1,3,4,7,12,12bhexahydro-13-hydroxy methyl-2H,6H-2,6-methano-indolo [2,3- $\alpha]$-quinolizine 8. The 6-epi$\mathrm{N}_{\mathrm{a}}$-methyl gardneral 7 (2.22 g, $\left.6.6 \mathrm{mmol}\right)$ was dissolved in EtOH $(40 \mathrm{~mL})$, and $\mathrm{NaBH}_{4}(240 \mathrm{mg}$, $6.6 \mathrm{mmol}$ ) was added to the above solution in one portion at $0{ }^{\circ} \mathrm{C}$. The mixture was then stirred at $0{ }^{\circ} \mathrm{C}$ for $8 \mathrm{~h}$. The reaction mixture was diluted with $\mathrm{CH}_{2} \mathrm{Cl}_{2}(300 \mathrm{~mL})$ and poured into cold water $(50 \mathrm{~mL})$. The aq layer was extracted with additional $\mathrm{CH}_{2} \mathrm{Cl}_{2}(3 \times 80 \mathrm{~mL})$, and the combined organic layers were washed with brine $(80 \mathrm{~mL})$ and dried $\left(\mathrm{K}_{2} \mathrm{CO}_{3}\right)$. The solvent was removed under reduced pressure to afford the crude product, which was purified by chromatography on silica gel $\left(\mathrm{CH}_{2} \mathrm{Cl}_{2} / \mathrm{CH}_{3} \mathrm{OH}=10: 1\right)$ to provide the 17-hydroxy derivative $8(2.12 \mathrm{~g}, 95 \%)$. ${ }^{1} \mathrm{H}$ NMR $\left(300 \mathrm{MHz}, \mathrm{CDCl}_{3}\right) \delta 1.64(3 \mathrm{H}, \mathrm{d}, \mathrm{J}=6.5 \mathrm{~Hz}), 1.66(\mathrm{~m}, 1 \mathrm{H}), 1.80(1 \mathrm{H}, \mathrm{q}, \mathrm{J}=7.2 \mathrm{~Hz}), 2.06$ (m, 1H), $2.59(1 \mathrm{H}, \mathrm{d}, \mathrm{J}=15.4 \mathrm{~Hz}), 2.74-2.81(\mathrm{~m}, 2 \mathrm{H}), 3.03(1 \mathrm{H}, \mathrm{dd}, \mathrm{J}=15.3,5.2 \mathrm{~Hz}), 3.46-3.65$ 
(m, 4H), $3.60(\mathrm{~s}, 3 \mathrm{H}), 3.90(\mathrm{~s}, 1 \mathrm{H}), 4.18(1 \mathrm{H}, \mathrm{d}, \mathrm{J}=8.30 \mathrm{~Hz}), 5.40(1 \mathrm{H}, \mathrm{q}, \mathrm{J}=6.5 \mathrm{~Hz}), 6.78(\mathrm{~m}$,

2H), $7.33(1 \mathrm{H}, \mathrm{d}, \mathrm{J}=8.3 \mathrm{~Hz}, 1 \mathrm{H}) ;{ }^{13} \mathrm{C} \mathrm{NMR}\left(75.5 \mathrm{MHz}, \mathrm{CDCl}_{3}\right) \delta$ 12.7, 27.0, 27.5, 29.3, 32.9, $44.2,49.4,54.2,55.8,56.3,65.0,93.1,103.4,108.0,116.4,118.5,121.8,136.1,136.9,137.9$, 155.8. EIMS(70eV) (m/e relative intensity): 338( $\left.\mathrm{M}^{+}, 83\right), 337(84), 213(100), 212(90)$. This material was employed directly in the next step.

Conversion of 16-Epi- $\mathrm{N}_{\mathrm{a}}$-methyl-17-hydroxygardnerine $8 \quad$ into $13-$ (Triisopropylsilanyloxymethyl)-3-ethylidene-10-methoxy-12-methyl-1,3,4,7,12,12b-

hexahydro-13-hydroxymethyl-2H,6H-2,6-methano-indolo[2,3- $\alpha]$-quinolizine 9. A solution of $8(1.32 \mathrm{~g}, 3.9 \mathrm{mmol})$ in dry $\mathrm{CH}_{2} \mathrm{Cl}_{2}(40 \mathrm{~mL})$ was cooled to $0{ }^{\circ} \mathrm{C}$, after which 2, 6-lutidine (1.8 $\mathrm{mL}, 15.6 \mathrm{mmol})$ was added, and this was followed by addition of TIPSOTf $(2.1 \mathrm{~mL}, 7.8 \mathrm{mmol})$ to the stirred solution. The mixture was then allowed to stir for an additional 2 hours at $0{ }^{\circ} \mathrm{C}$, after which cold water $(2 \mathrm{~mL})$ was added to quench the reaction mixture. The reaction mixture was diluted with $\mathrm{CH}_{2} \mathrm{Cl}_{2}(100 \mathrm{~mL})$ and poured into cold water $(20 \mathrm{~mL})$. The aq layer was extracted with additional $\mathrm{CH}_{2} \mathrm{Cl}_{2}(2 \times 50 \mathrm{~mL})$, and the combined organic layer was washed with brine $(30 \mathrm{~mL})$ and dried $\left(\mathrm{Na}_{2} \mathrm{SO}_{4}\right)$. The solvent was removed under reduced pressure to afford the crude product, which was dried in vacuo to remove the excess 2,6-lutidine before the solid was purified by chromatography on silica gel $\left(\mathrm{CH}_{2} \mathrm{Cl}_{2} / \mathrm{CH}_{3} \mathrm{OH}=20: 1\right)$ to provide the O-TIPS ether $9(1.74 \mathrm{~g})$ in $90 \%$ yield. ${ }^{1} \mathrm{H}$ NMR $\left(\mathrm{CDCl}_{3}, 300 \mathrm{MHz}\right): \delta 1.06(21 \mathrm{H}, \mathrm{m}), 1.65(3 \mathrm{H}, \mathrm{d}, \mathrm{J}=6.8$ $\mathrm{Hz}), 1.78(1 \mathrm{H}, \mathrm{m}), 1.93(1 \mathrm{H}, \mathrm{q}, \mathrm{J}=7.2 \mathrm{~Hz}), 2.16(1 \mathrm{H}, \mathrm{t}, \mathrm{J}=11.9 \mathrm{~Hz}), 2.71(1 \mathrm{H}, \mathrm{d}, \mathrm{J}=15.7 \mathrm{~Hz})$, 2.86-2.89(2H, m), 3.20(1H, dd, J = 15.4, $4.6 \mathrm{~Hz}), 3.58(3 \mathrm{H}, \mathrm{s}), 3.60(2 \mathrm{H}, \mathrm{m}), 3.74(2 \mathrm{H}, \mathrm{br})$, $3.90(3 \mathrm{H}, \mathrm{s}), 4.36(1 \mathrm{H}, \mathrm{d}, \mathrm{J}=9.5 \mathrm{~Hz}), 5.47(1 \mathrm{H}, \mathrm{q}, \mathrm{J}=6.8 \mathrm{~Hz}), 6.77(2 \mathrm{H}, \mathrm{m}), 7.35(1 \mathrm{H}, \mathrm{d}, \mathrm{J}=8.0$ $\mathrm{Hz}) ;{ }^{13} \mathrm{C} \mathrm{NMR}\left(\mathrm{CDCl}_{3}, 75.5 \mathrm{MHz}\right) \delta 11.8,12.8,18.0,25.4,27.1,29.3,32.9,44.2,49.5,54.9$, 55.8, 56.3, 65.5, 93.1, 103.5, 108.0, 116.6, 118.6, 121.8, 135.3, 137.9, 138.0, 155.8; EIMS(70eV, 
m/e relative intensity): 494( $\left.\mathrm{M}^{+}, 11\right), 307(36), 213(78), 212(100)$. Anal. Calcd. for $\mathrm{C}_{30} \mathrm{H}_{46} \mathrm{~N}_{2} \mathrm{O}_{2} \mathrm{Si}$ : C, 72.82\%; H, 9.37\%; N, 5.66\%. Found: C, 72.59\%; H, 9.02\%; N, 5.85\%.

\section{Preparation of 1-[13-(Triisopropylsilanyloxymethyl)-3-ethylidene-10-methoxy-12-methyl-} 1,3,4,7,12,12b-hexahydro-2H,6H-methano-indolo[2,3- $\alpha]$-quinolizine-3-yl]-ethanol (11a) and

(11b). To a solution of triisopropylsilyl ether $9(531 \mathrm{mg}, 1.08 \mathrm{mmol})$ in dry THF (24 mL) was added $\mathrm{BH}_{3} \bullet \mathrm{DMS}(1 \mathrm{M}, 10.0 \mathrm{~mL}, 10.0 \mathrm{mmol})$ at $\mathrm{rt}$. The mixture which resulted was stirred at $\mathrm{rt}$ for $3 \mathrm{~h}$. The reaction mixture was then quenched by careful addition of water $(4.7 \mathrm{~mL})$ at $0{ }^{\circ} \mathrm{C}$. At this point aq $\mathrm{NaOH}(3 \mathrm{~N}, 20 \mathrm{~mL}, 60 \mathrm{mmol})$ was added to the mixture followed by addition of $\mathrm{H}_{2} \mathrm{O}_{2}(30 \%, 5.0 \mathrm{~mL}, 43 \mathrm{mmol})$. The mixture which resulted was allowed to stir at $\mathrm{rt}$ for $2 \mathrm{~h}$ after which EtOAc $(400 \mathrm{~mL})$ and $\mathrm{H}_{2} \mathrm{O}(50 \mathrm{~mL})$ were added. The organic layer was separated and dried $\left(\mathrm{Na}_{2} \mathrm{SO}_{4}\right)$. The EtOAc was then removed under reduced pressure and the residue was purified by flash chromatography with EtOAc/hexanes (3:7) to provide a mixture of isomers at [C(19)] 10a and 10b $(511 \mathrm{mg})$ in $90 \%$ yield. The $\mathrm{BH}_{3}$ complex $10 \mathrm{a}$ was isolated as the major product: ${ }^{1} \mathrm{H}$ NMR $\left(300 \mathrm{MHz}, \mathrm{CDCl}_{3}\right) \delta 1.02-1.09(21 \mathrm{H}, \mathrm{m}), 1.20(3 \mathrm{H}, \mathrm{d}, \mathrm{J}=5.8 \mathrm{~Hz}), 1.71-$ $1.81(2 \mathrm{H}, \mathrm{m}), 2.08(1 \mathrm{H}, \mathrm{q}, \mathrm{J}=9.2 \mathrm{~Hz}), 2.18(1 \mathrm{H}, \mathrm{t}, \mathrm{J}=10.7 \mathrm{~Hz}), 2.53-2.57(2 \mathrm{H}, \mathrm{m}), 2.83(1 \mathrm{H}, \mathrm{m})$, $2.89(1 \mathrm{H}, \mathrm{dd}, \mathrm{J}=14.7,5.3 \mathrm{~Hz}), 3.48(1 \mathrm{H}, \mathrm{dd}, \mathrm{J}=15.5,4.9 \mathrm{~Hz}), 3.57(1 \mathrm{H}, \mathrm{m}), 3.60(3 \mathrm{H}, \mathrm{s}), 3.79-$ 3.87(2H, m), 3.90(3H, s), 4.01(1H, dd, J = 11.0, 7.6 Hz), 4.22(1H, d, J = 9.3 Hz), 6.78(2H, m), 7.34(1H, d, J = 9.3 Hz); ${ }^{13} \mathrm{C} \mathrm{NMR}\left(75.5 \mathrm{MHz}, \mathrm{CDCl}_{3}\right) \delta 11.8,17.9,21.8,22.6,26.8,29.6,36.5$, $42.1,45.5,54.9,55.9,60.3,61.2,65.8,71.5,93.3,101.8,108.6,118.9,121.2,136.4,138.2$ 156.1; EIMS (70eV, m/e, relative intensity) 512( $\left.\mathrm{M}^{+}, 1.4\right), 267(19), 226(34), 213(60), 212(100)$. This material was used in the next step without further purification. The above mixture of isomers (506 mg, $0.9 \mathrm{mmol})$ was dissolved in $\mathrm{MeOH}(30 \mathrm{~mL})$, and $\mathrm{Na}_{2} \mathrm{CO}_{3}(477.0 \mathrm{mg}, 4.5$ 
mmol) was added. The mixture was then warmed to reflux overnight. The reaction mixture which resulted was cooled to rt followed by filtration to remove solids and after which it was diluted with EtOAc $(200 \mathrm{~mL})$. The solution which resulted was then washed with water $(30 \mathrm{~mL})$ and brine $(50 \mathrm{~mL})$. The solvent was removed under reduced pressure to afford a crude solid, which was purified by chromatography [silica gel, $\mathrm{CH}_{2} \mathrm{Cl}_{2} / \mathrm{MeOH}$ (v/v 10:1)] to provide the mixtures of sec-ols (11a) and (11b) $(424 \mathrm{mg}, 0.83 \mathrm{mmol})$ in $92 \%$ yield. 11a: ${ }^{1} \mathrm{H}$ NMR $(300$ $\left.\mathrm{MHz}, \mathrm{CDCl}_{3}\right) \delta 1.02-1.09(21 \mathrm{H}, \mathrm{m}), 1.18(3 \mathrm{H}, \mathrm{d}, \mathrm{J}=5.9 \mathrm{~Hz}), 1.52(2 \mathrm{H}, \mathrm{dt}, \mathrm{J}=12.2,3.4 \mathrm{~Hz}), 1.84-$ $1.94(2 \mathrm{H}, \mathrm{m}), 2.46(1 \mathrm{H}, \mathrm{bs}), 2.48(1 \mathrm{H}, \mathrm{d}, \mathrm{J}=15.2 \mathrm{~Hz}), 2.62(1 \mathrm{H}, \mathrm{dd}, \mathrm{J}=14.1,5.1 \mathrm{~Hz}), 2.78(1 \mathrm{H}$, $\mathrm{dd}, \mathrm{J}=6.5,5.8 \mathrm{~Hz}), 3.07(1 \mathrm{H}, \mathrm{dd}, \mathrm{J}=15.3,5.4 \mathrm{~Hz}), 3.34(1 \mathrm{H}, \mathrm{dd}, \mathrm{J}=14.1,10.5 \mathrm{~Hz}), 3.60(3 \mathrm{H}$, s), 3.78-3.86 (2H, m), $3.90(3 \mathrm{H}, \mathrm{s}), 4.00(1 \mathrm{H}, \mathrm{dd}, \mathrm{J}=10.9,7.5 \mathrm{~Hz}), 4.12(1 \mathrm{H}, \mathrm{dd}, \mathrm{J}=9.3,2.1$ $\mathrm{Hz}), 6.75-6.80(2 \mathrm{H}, \mathrm{m}), 7.35(1 \mathrm{H}, \mathrm{d}, \mathrm{J}=8.4 \mathrm{~Hz}) ;{ }^{13} \mathrm{C} \mathrm{NMR}\left(75.5 \mathrm{MHz}, \mathrm{CDCl}_{3}\right) \delta 11.8,17.9$, 21.5, 22.6, 27.7, 29.2, 37.2, 41.8, 44.0, 48.7, 53.3, 55.4, 55.7, 66.5, 72.0, 93.1, 102.6, 107.9, 118.5, 121.8, 137.9, 138.7, 155.7. 11b: ${ }^{1} \mathrm{H}$ NMR (300 $\left.\mathrm{MHz}, \mathrm{CDCl}_{3}\right) \delta 1.02-1.09(21 \mathrm{H}, \mathrm{m})$, $1.28(3 \mathrm{H}, \mathrm{d}, \mathrm{J}=6.0 \mathrm{~Hz}), 1.48-1.55(2 \mathrm{H}, \mathrm{m}), 1.75(1 \mathrm{H}, \mathrm{q}, \mathrm{J}=7.6 \mathrm{~Hz}), 1.87(1 \mathrm{H}, \mathrm{t}, \mathrm{J}=10.8 \mathrm{~Hz})$, $2.01(1 \mathrm{H}, \mathrm{bs}), 2.71(1 \mathrm{H}, \mathrm{d}, \mathrm{J}=14.9 \mathrm{~Hz}), 2.96-3.06(3 \mathrm{H}, \mathrm{m}), 3.27-3.32(1 \mathrm{H}, \mathrm{m}), 3.43(3 \mathrm{H}, \mathrm{s})$, 3.60-3.65 (1H, m), 3.72-3.75 (1H, m), 3.86-3.91 (1H, m), $3.90(2 \mathrm{H}, \mathrm{s}), 4.01(1 \mathrm{H}, \mathrm{dd}, \mathrm{J}=9.7,1.7$ $\mathrm{Hz}), 6.75-6.78(2 \mathrm{H}, \mathrm{m}), 7.33(1 \mathrm{H}, \mathrm{d}, \mathrm{J}=8.1 \mathrm{~Hz}) ;{ }^{13} \mathrm{C} \mathrm{NMR}\left(75.5 \mathrm{MHz}, \mathrm{CDCl}_{3}\right) \delta 11.8,17.9$, $22.2,25.7,27.4,29.1,38.0,42.7,44.2,48.6,54.0,55.6,55.7,66.2,71.7,93.0,102.8,108.0$, $118.6,121.8,137.8,138.0,155.7$. This material was employed directly in the next step.

IBX Mediated Oxidation of the Sec-ols 11a and 11b to Provide the 1-[13(Triisopropylsilanyloxymethyl)-3-ethylidene-10-methoxy-6-oxo-12-methyl-1,3,4,7,12,12bhexahydro-2H,6H-methano-indolo[2,3- $\alpha]$-quinolizine-3-yl]-ethanone (13). To a solution of the sec-ols (11a and 11b, $425.6 \mathrm{mg}, 0.83 \mathrm{mmol})$ in EtOAc/DMSO (30 mL/15 mL)) was added 
IBX (929 mg, $3.32 \mathrm{mmol}$ ) in one portion at $\mathrm{rt}$. The mixture which resulted was then stirred at 80 ${ }^{\circ} \mathrm{C}$ for $8 \mathrm{~h}$ and the reaction progress was monitored by TLC (silica gel, EtOAc)]. The reaction mixture was cooled to $0{ }^{\circ} \mathrm{C}$ and was quenched with a saturated solution of aq $\mathrm{NaHCO}_{3}(9 \mathrm{~mL})$, followed by treatment with a saturated solution of aq $\mathrm{Na}_{2} \mathrm{~S}_{2} \mathrm{O}_{3}(9 \mathrm{~mL})$ after which the mixture was stirred for an additional $10 \mathrm{~min}$ at $0{ }^{\circ} \mathrm{C}$. The aq layer was extracted with additional amounts of EtOAc $(3 \times 30 \mathrm{~mL})$, and the combined organic layer was washed with brine $(20 \mathrm{~mL})$ and dried $\left(\mathrm{K}_{2} \mathrm{CO}_{3}\right)$. The solvent was removed under reduced pressure to provide the crude oil which was purified by chromatography [silica gel, $\mathrm{CH}_{2} \mathrm{Cl}_{2} / \mathrm{MeOH}$ (v/v 20:1)] to provide the desired ketone $13(370.2 \mathrm{mg}, 85 \%) .{ }^{1} \mathrm{H}$ NMR $\left(300 \mathrm{MHz}, \mathrm{CDCl}_{3}\right) \delta 1.07-1.10(21 \mathrm{H}, \mathrm{m}), 1.38(1 \mathrm{H}, \mathrm{d}, \mathrm{J}=5.4 \mathrm{~Hz})$, 1.98-2.07 (2H, m), $2.23(3 \mathrm{H}, \mathrm{s}), 2.72(1 \mathrm{H}, \mathrm{bs}), 2.99-3.17(3 \mathrm{H}, \mathrm{m}), 3.64(3 \mathrm{H}, \mathrm{s}), 3.70(1 \mathrm{H}, \mathrm{dd}, \mathrm{J}=$ 13.4, 5.7 Hz), $3.84(1 \mathrm{H}, \mathrm{d}, \mathrm{J}=10.0 \mathrm{~Hz}), 3.89(3 \mathrm{H}, \mathrm{s}), 4.04(1 \mathrm{H}, \mathrm{dd}, \mathrm{J}=10.5,4.5 \mathrm{~Hz}), 4.25(1 \mathrm{H}$, dd, J = 10.1, $3.7 \mathrm{~Hz}), 6.80(1 \mathrm{H}, \mathrm{d}, \mathrm{J}=1.6 \mathrm{~Hz}), 6.94(1 \mathrm{H}, \mathrm{dd}, \mathrm{J}=8.6,1.5 \mathrm{~Hz}), 8.02(1 \mathrm{H}, \mathrm{d}, \mathrm{J}=8.6$ $\mathrm{Hz}) ;{ }^{13} \mathrm{C} \mathrm{NMR}\left(75.5 \mathrm{MHz}, \mathrm{CDCl}_{3}\right) \delta 11.8,18.0,27.9,29.0,29.1,30.1,40.8,42.0,47.6,48.8$, $55.7,63.1,63.8,94.1,105.6,111.3,118.4,122.3,138.5,155.3,157.1,192.2,210.0$. This material was employed directly in the next step.

\section{$\mathrm{N}_{\mathrm{b}}$-Methylation of Ketone 13 Followed by Base Mediated Retro-Michael Reaction to} Provide the 11-Methoxy-6-oxo-macroline Equivalent 14. To a solution of ketone 13 (525 mg, $1.0 \mathrm{mmol})$ in THF $(25 \mathrm{~mL})$, MeI $(212.9 \mathrm{mg}, 1.5 \mathrm{mmol})$ was added at $0{ }^{\circ} \mathrm{C}$, after which the mixture was allowed to stir in the dark at $0{ }^{\circ} \mathrm{C}$ overnight. The solvent was then removed under reduced pressure and the residue was dissolved in THF (40 mL) after which $\mathrm{K}_{2} \mathrm{CO}_{3}(691 \mathrm{mg}, 5.0$ mmol)) was added. The mixture which resulted was allowed to heat to reflux for $12 \mathrm{hr}$ after which it was cooled to rt. The EtOAc $(600 \mathrm{~mL})$ and $\mathrm{H}_{2} \mathrm{O}(70 \mathrm{~mL})$ were then added and the 
organic layer was washed with brine $(70 \mathrm{~mL})$ and dried $\left(\mathrm{Na}_{2} \mathrm{SO}_{4}\right)$. The EtOAc was removed under reduced pressure and the residue was purified by flash chromatography [silica gel, EtOAc/hexane (v/v 1:1)] to provide 14 (485 mg, $0.9 \mathrm{mmol})$ in $90 \%$ yield. ${ }^{1} \mathrm{H}$ NMR (300 MHz, $\left.\mathrm{CDCl}_{3}\right) \delta 1.06(21 \mathrm{H}, \mathrm{m}), 1.50(1 \mathrm{H}, \mathrm{m}), 2.12(1 \mathrm{H}, \mathrm{m}), 2.24(3 \mathrm{H}, \mathrm{s}), 2.37(1 \mathrm{H}, \mathrm{m}), 2.46(3 \mathrm{H}, \mathrm{s})$, $3.05(1 \mathrm{H}, \mathrm{m}), 3.57(1 \mathrm{H}, \mathrm{br}), 3.89(3 \mathrm{H}, \mathrm{s}), 3.92-3.95(1 \mathrm{H}, \mathrm{m}), 4.06(1 \mathrm{H}, \mathrm{m}), 4.21(1 \mathrm{H}, \mathrm{br}), 5.60$ $(1 \mathrm{H}, \mathrm{s}), 6.00(1 \mathrm{H}, \mathrm{s}), 6.81(1 \mathrm{H}, \mathrm{d}, \mathrm{J}=2.2 \mathrm{~Hz}), 6.96(1 \mathrm{H}, \mathrm{dd}, \mathrm{J}=8.6,2.2 \mathrm{~Hz}), 8.14(1 \mathrm{H}, \mathrm{d}, \mathrm{J}=8.6$ $\mathrm{Hz}) ;{ }^{13} \mathrm{C} \mathrm{NMR}\left(\mathrm{CDCl}_{3}, 75.5 \mathrm{MHz}\right) \delta 11.9,17.9,26.3,28.0,29.6,29.8,40.4,42.9,54.1,55.8$, $60.3,66.2,94.0,108.8,111.2,117.9,122.6,123.0,138.5,146.8,150.2,157.0,193.9,198.4$. This material was employed directly in the next step.

(-)-Alstonerine 16. To a solution of the macroline equivalent 15 (41 mg, 0.083mmol)in AcOH/ $\mathrm{H}_{2} \mathrm{O} / \mathrm{tBuOH}(0.6 \mathrm{~mL} / 1.8 \mathrm{~mL} / 1.8 \mathrm{~mL})$ were added $\mathrm{Na}_{2} \mathrm{PdCl}_{4}(29.5 \mathrm{mg}, 0.10 \mathrm{mmol}), \mathrm{NaOAc}(6.8$ $\mathrm{mg}, 0.083 \mathrm{mmol})$ and $\mathrm{TBHP}(0.014 \mathrm{~mL})$ at $\mathrm{rt}$ and the mixture which resulted was stirred at $80{ }^{\circ} \mathrm{C}$ for $6 \mathrm{hr}$. After the mixture was cooled to rt, ice water $(2 \mathrm{~mL})$ and a sat solution of aq $\mathrm{NaHCO}_{3}(10$ $\mathrm{mL})$ were added to the mixture and this was followed by addition of $\operatorname{EtOAc}(50 \mathrm{~mL})$. The aq layer was extracted with additional EtOAc $(2 \times 30 \mathrm{~mL})$. The combined organic layer was washed with a saturated solution of aq $\mathrm{NaHCO}_{3}$, brine, and dried $\left(\mathrm{K}_{2} \mathrm{CO}_{3}\right)$. The EtOAc was removed under reduced pressure and the residue was purified by preparative TLC [silica gel,EtOAc/hexane(v/v1:1)] to provide $16(12.5 \mathrm{mg}, 0.034 \mathrm{mmol})$ in $60 \%$ yield. ${ }^{1} \mathrm{H}$ NMR (300 $\left.\mathrm{MHz}, \mathrm{CDCl}_{3}\right) \delta 1.82(1 \mathrm{H}, \mathrm{dd}, \mathrm{J}=12.2,4.2 \mathrm{~Hz}), 1.92(1 \mathrm{H}, \mathrm{ddd}, \mathrm{J}=12.2,11.4,1.5 \mathrm{~Hz}), 2.11(3 \mathrm{H}$, s), 2.11-2.19 (1H, m), $2.35(3 \mathrm{H}, \mathrm{s}), 2.53(1 \mathrm{H}, \mathrm{d}, \mathrm{J}=16.5 \mathrm{~Hz}), 2.64(1 \mathrm{H}, \mathrm{dt}, \mathrm{J}=12.4,4.6 \mathrm{~Hz}), 3.11$ $(1 \mathrm{H}, \mathrm{d}, \mathrm{J}=6.8 \mathrm{~Hz}), 3.36(1 \mathrm{H}, \mathrm{dd}, \mathrm{J}=16.4,6.8 \mathrm{~Hz}), 3.68(3 \mathrm{H}, \mathrm{s}), 3.90(1 \mathrm{H}, \mathrm{t}, \mathrm{J}=1.5 \mathrm{~Hz}), 4.19$ $(1 \mathrm{H}, \mathrm{ddd}, \mathrm{J}=11.2,4.0,1.5 \mathrm{~Hz}), 4.44(1 \mathrm{H}, \mathrm{t}, \mathrm{J}=11.2 \mathrm{~Hz}), 7.11(1 \mathrm{H}, \mathrm{t}, \mathrm{J}=8.1 \mathrm{~Hz}), 7.22(1 \mathrm{H}, \mathrm{t}, \mathrm{J}=$ 
$7.0 \mathrm{~Hz}), 7.34(1 \mathrm{H}, \mathrm{d}, \mathrm{J}=8.1 \mathrm{~Hz}), 7.50(1 \mathrm{H}, \mathrm{d}, \mathrm{J}=7.6 \mathrm{~Hz}), 7.55(1 \mathrm{H}, \mathrm{s}) ;{ }^{13} \mathrm{C} \mathrm{NMR}(75 \mathrm{MHz}$, $\left.\mathrm{CDCl}_{3}\right) \delta 22.7,22.8,24.9,29.0,32.3,38.4,41.7,53.7,54.6,67.7,105.8,108.9,117.7,118.6$, 120.7, 121.0, 126.5, 133.1, 137.1, 157.3, 195.4. IR (NaCl) 1650, $1621 \mathrm{~cm}^{-1}$. EIMS (m/e, relative intensity) $336\left(\mathrm{M}^{+}\right.$, 47), 197 (100), 182 (58), 181 (82), 170 (89). HRMS: calcd. for $\mathrm{C}_{21} \mathrm{H}_{24} \mathrm{~N}_{2} \mathrm{O}_{2}$ : 336.1846, found 336.1838. The spectral data were in excellent agreement with those of natural alstonerine. ${ }^{1}$

(+)-6-Oxoalstophylline 1. The synthesis of $\mathbf{1}$ from $\mathbf{1 4}$ was carried out analogous to the preparation of (-)-alstonerine 16 from 15 in $57 \%$ yield. ${ }^{1} \mathrm{H} \mathrm{NMR}\left(500 \mathrm{MHz}, \mathrm{CDCl}_{3}\right) \delta 1.97(\mathrm{~m}$, 1H), $2.11(\mathrm{~s}, 3 \mathrm{H}), 2.20(\mathrm{~m}, 2 \mathrm{H}), 2.44(\mathrm{~s}, 3 \mathrm{H}), 2.70(\mathrm{~m}, 1 \mathrm{H}), 3.09(\mathrm{~s}, 1 \mathrm{H}), 3.64(\mathrm{~s}, 3 \mathrm{H}), 3.91(\mathrm{~s}, 3 \mathrm{H})$, 4.05(br, 1H), $4.25(\mathrm{ddd}, \mathrm{J}=11,4,1.6 \mathrm{~Hz}, 1 \mathrm{H}), 4.45(\mathrm{t}, \mathrm{J}=11 \mathrm{~Hz}, 1 \mathrm{H}), 6.85(\mathrm{~d}, \mathrm{~J}=2 \mathrm{~Hz}, 1 \mathrm{H})$, $6.96(\mathrm{dd}, \mathrm{J}=8.5,2.2 \mathrm{~Hz}, 1 \mathrm{H}), 7.56(\mathrm{~s}, 1 \mathrm{H}), 8.04(\mathrm{~d}, \mathrm{~J}=8.5 \mathrm{~Hz}, 1 \mathrm{H}) . \mathrm{IR}(\mathrm{NaCl}) 1645,1616 \mathrm{~cm}^{-1}$. EIMS (m/e, relative intensity) $380\left(\mathrm{M}^{+}, 52\right), 350$ (5), 293 (10), 255 (27), 214 (100), 175 (9), 111 (20), 70 (64). The spectra data were in excellent agreement with those of the natural product $\mathbf{1}^{2}$

(-)-Alstophylline 2. The synthesis of $\mathbf{2}$ from $\mathbf{1 7}$ was carried out analogous to the preparation of ()-alstonerine 16 from 15 in 55\% yield. ${ }^{1} \mathrm{H}$ NMR $\left(300 \mathrm{MHz}, \mathrm{CDCl}_{3}\right) 1.81(1 \mathrm{H}, \mathrm{m}), 1.91(1 \mathrm{H}, \mathrm{m})$, $2.09(3 \mathrm{H}, \mathrm{s}), 2.32(3 \mathrm{H}, \mathrm{bs}), 2.45(1 \mathrm{H}, \mathrm{bd}, \mathrm{J}=16.5 \mathrm{~Hz}), 2.61(1 \mathrm{H}, \mathrm{m}), 3.07(1 \mathrm{H}, \mathrm{m}), 3.29(1 \mathrm{H}, \mathrm{m})$, $3.59(3 \mathrm{H}, \mathrm{s}), 3.83(1 \mathrm{H}, \mathrm{bs}), 3.89(3 \mathrm{H}, \mathrm{s}), 4.16(1 \mathrm{H}, \mathrm{dd}, \mathrm{J}=11.2,2.3 \mathrm{~Hz}), 4.43(1 \mathrm{H}, \mathrm{t}, \mathrm{J}=11.2 \mathrm{~Hz})$, $6.75(1 \mathrm{H}, \mathrm{dd}, \mathrm{J}=8.5,2.0 \mathrm{~Hz}), 6.80(1 \mathrm{H}, \mathrm{d}, \mathrm{J}=1.8 \mathrm{~Hz}), 7.33(1 \mathrm{H}, \mathrm{d}, \mathrm{J}=8.4 \mathrm{~Hz}), 7.53(1 \mathrm{H}, \mathrm{s})$. EIMS (m/e) $366\left(\mathrm{M}^{+}\right), 227,212,211,200$. The spectra data were in excellent agreement with those of natural alstophylline. ${ }^{3}$ 


\section{References:}

1. Elderfield, R. C.; Gilman, R. E. Phytochemistry 1972, 11, 339.

2. Kam, T.S.; Choo, Y.M. J. Nat. Prod. 2004, 67, 547.

3. a) Kishi, T.; Hesse, M.; Gemenden, C.W.; Taylor, W.I.; Schmid, H. Helv. Chim. Acta, 1965, 48, 1349. b) Abe, F.; Yamauchi, T.; Santisuk, T. Phytochemistry, 1994, 35, 249252. 